\title{
AN EXPERIMENTAL COURSE ON DIGITAL COMMUNICATIONS
}

\author{
Bertran, E.; Tarres, F.; Montoro, G. \\ Polytechnic University of Catalonia \\ Department of Signal Theory and Communications. \\ Campus Nord UPC, Modul D4. C/ Jordi Girona 1-3 \\ 08034 . Barcelona (Spain) \\ email: bertran@tsc.upc.es
}

\begin{abstract}
In this paper a laboratory course on digital communications is presented. This course has been designed for medium degree professionals in the telecommunications field, and it is based on a trainer equipment developed to change the usual theoretical classrooms for laboratory seminars.
\end{abstract}

\section{1.- INTRODUCTION.}

During the last two decades the analog communications have transferred part of its protagonism to the digital ones. Digital communication systems were limited to specific applications at their beginning, being these applications reserved to specialized professionals. But nowadays the medium degree professionals in the telecommunications field must also to work with digital communications systems. The proliferation in the market of products such as modems, digital telephony subsystems, recorders and reproducers for digital audio and video, satellite television receivers, or new systems such as the DAB and DVB, evidence the needs of practical formation in digital communications for professional communities up to now little introduced in them.

The traditional formation of the medium degree professionals -in the telecommunication areaincludes, among other aspects, analog electronics, digital circuits and analog modulations. In these subjects they receive theoretical formation and practice. It doesn't happen the same with the digital communications, where their formation is, as much, theoretical and in a merely introductory level [1].

It is no new to educate students with good mathematical bases in topics such as the random variables or the Fourier transform in digital communications. This is the case of the engineering university students. But to form students of other communities, more guided to the installation, maintenance and repair than to the theoretical design and development, it is necessary to teach with more limited scientific tools. Due to their future professional orientation, these students receive a formation very addressed to the practice, what seems correct for the society objectives. Any intent of augmentation of the mathematical bases would necessarily affect or in a delay of its period of scholarly, or in the distortion of their applied knowledge. Both situations are undesirable since they would suppose to change their professional profile, lengthening their incorporation to the work market.

In this paper a 45 hours laboratory course is presented. This course is thought to introduce, from a practical point of view, the bases of the digital communications to the students of medium professional degree. These bases covers from the base-band transmission to the continuous carrier digital modulations. The final objective is the learning of the advantages and restrictions of the main digital modulations [2], as well as electronic circuits useful in their development. This course has been developed by university teachers, in collaboration with teachers of half degree schools. So, it combines the systems engineering point of view with the repair and maintenance one. The task has not been easy, since the development of some aspects has required a wide study and a didactic reflection previous to its translation to a basic language or to simple circuit realizations.

\section{2.- THE STUDENTS' PROFILE.}

The design of this experimental course starts from the previous level of the students, based on the disciplines that at present are imparted in telecommunication and electronics professional formation. Therefore, the departure bases will be some knowledge in:

- Analog electronics: basic assemblies with passive components, bipolar transistors and elementary applications with operational amplifiers.

- Digital electronics: development of combinatory and sequential circuits with discrete components. 
Previous bases in programming of microprocessors are not required, neither of ASIC circuits.

- Analog communications: some elementary knowledge of the amplitude and frequency modulations are supposed.

On the other hand, we assume a certain psychological profile of the students, those we will consider more motivated by the applications than for theory, and with more easiness for the experimental learning than for the merely theoretical one.

\section{3.- DESIGN OF A TRAINER EQUIPMENT FOR DIGITAL COMMUNICATIONS.}

In order to support the practices of the course, a trainer of digital communications has been developed [3]. It consists on two modules: the transmitter and the receiver. Due to the characteristics of the course and those of the students, the whole trainer has been developed with discrete components, without microprocessors or ASIC developments. This fact has a double objective: on one hand the students can analyze the different blocks (circuits) without significant deviations regarding their basic formation in electronics; on the other hand all the signals of interest in the subsystems are accessible (observables in a oscilloscope). The use of more advanced digital components should facilitate the trainer's design, but at the price of hiding didactic interest signals. The equipment includes the following subsystems, where $[E]$ and $[R]$ indicate if they are located in the emitter module or in the receiver:

[E] Sampling and acquisition of analog signals.This block is formed by the antialiasing filter, an analog-to digital (A/D) flash conversor, a signal compressor and a UART for serial transmission (pulse code modulation, PCM). In this subsystem one can experience the effect of the antialiasing filter $\left(8^{\text {th }}\right.$ order low-pass filter) and that of the compressor (analog) so much with microphone inputs as with function generators.

[R] Reconstruction of analog signals.- In this block the output of the receiver UART is addressed to an D/A conversor, and then passed through a reconstruction filter (correction of the aperture distortion) and an analog expander. The output can be observed in an oscilloscope or listened in the speakers. In this part the effects of the number of bits used in the analog signal coding are studied, as well as those of the reconstruction filter and the expander (an approximate gain of $20 \mathrm{~dB}$ in the reconstructed signal).
[E][R] Transmission in base band.- It allows to connect directly the output of the transmitter UART to the input of the receiver UART (Basic PCM transmission)

[R] Modulation and [R] demodulation in ASK.- The emitter is based on a switched Colpitts oscillator, and the receiver on a band-pass filter with an envelope detector.

[E] Modulation and [R] demodulation in FSK.- The emitter is a continuous phase VCO. For reception, they can be selected a block based on a PLL or another block with a dual filter detector (DFD) in order to compare their performances.

[E] Modulation and [R] demodulation in BPSK.- In this block the modulation is made with another Colpitts oscillator. An analog multiplexer controlled by the PCM signal selects the in-phase output of the oscillator or the inverted phase output. The demodulation is based on the product of a reconstructed carrier with the modulated input. The product is made with a XNOR CMOS gate.

[E] Modulation and [R] demodulation in DPSK.- The modulator in differential phase shift keying is based on the BPSK one, where the PCM signal is previously differentially coded though a EX-NOR gate. The demodulator is also the same of the BPSK one, with a previous differential decoder (CMOS).

[E] Modulation and [R] demodulation in QPSK.The dibits for quadrature modulation are obtained from a CMOS circuitry that translates the serial PCM signal to be modulated to two parallel dibits. These dibits controls a multiplexer that selects the corresponding phase. The $0^{\circ}, 90^{\circ}, 180^{\circ}$ and $270^{\circ}$ phases are obtained with a shift-register at the output of the oscillator. In demodulation, these reference phases are obtained from the reconstructed carrier, that is obtained with a nonlinear block and an analog PLL.

[E] Modulation and [R] demodulation in DQPSK.The -modulator is the same than the QPSK one, where previously the PCM signal has been differentially coded. The demodulator is also se same than in QPSK, with a previous differential decoder.

$[E]$ Modulation and [R] demodulation in QAM.- The PCM signal is previously converted in tribits with CMOS circuitry. Two tribits controls the phase like in the QPSK modulation, whereas the third tribit controls the output amplitude. In reception, the first block is an amplitude controller (AGC). The tribits are detected from a QPSK detector (two tribits) and an amplitude comparator (third tribit).

[E] [R] Communication channels.- The connection between the emitter module and the receiver one can be done through the following communication 
channel: twisted cable, coaxial, fiber optics, infrared or radio. This allows to experience and compare the behaviour of different digital modulations in front of these channels.

[E] Channel disturbances.- In order to study real communications problems in the course practices, a channel disturbances simulator has been added to the trainer. These disturbances include band-width (BW) reduction, pseudo-noises (PRN), interferences and channel attenuations.

In the development of some modulation and demodulation subsystems we tried do not disperse the learning objectives. In this way, some aspects related with protocols, administration of the communications and learning sequences have been obviated by means of the development of novel structures [4].

\section{4. - LABORATORY EXPERIENCES.}

The practices covers from basic aspects (and of capital importance) of sampling and quantification of analog signals for its later digital transmission (PCM), until all the basic digital modulations and demodulations. Deteriorated situations in the channel of communications in form of attenuations, noises and interferences are also experienced. The practices present also alternative aspects of visualization, such as the eye diagrams and the constellations. The use of audio subsystems is introduced early (microphone and headphones), in order to facilitate that interested students can enjoy during the whole laboratory course hearing different qualities of voice signal or tones generated from the functions generator and transmitted digitally.

They have been designed leaving of the habitual equipment in a laboratory of basic electronics, and it has been supposed that the oscilloscopes to be used in the practices are analog. They are not necessary more advanced oscilloscopes for them, although, in the case of having digital oscilloscopes, the visualization of some points of the practices will be more easy. And, in the scarce medium-degree laboratories where a spectra analyzer is available, it could be added to the temporary measurements their equivalent frequency visualizations.

The order of the practices is thought for people not begun in the digital communications field, so it is recommended to follow them strictly. The knowledge contributed in each practice grows from those of previous practices.
Practice 1. - Conversion A/D and D/A. Sampling and quantification.

The main objective of this practice is to take a first contact with the effects of the sampling and quantification of signals. In the first place experiments are made on oscilloscope signals, being visualized the effects of correct and incorrect samplings (Nyquist Theorem), as well as the form of the quantified signal. In second place, the effects of the quantification are listened, as well as the beneficial effect of the compander in vowel signals. Finally, some effects related to the reduction of the channel band-width and to the noises in the PCM transmitted signal are studied, evaluating the maximum base-band speed in the different cases. Sampling theorem is also studied, experimenting the sampling of sinusoids with and without the antialiasing filter.

The theoretical bases for the correct understanding and realization of this practice are sampling, quantification and PCM coding. These concepts are taught in a previous 4 hour seminar. Optionally, some technological aspects associated to this practice can be studied in electronic courses. Some of these aspects are: conversion $\mathrm{A} / \mathrm{D}$ and $\mathrm{D} / \mathrm{A}$, characteristics of the $A / D, D / A$ and expander devices, and UART operation.

Practice 2.- ASK modulation and demodulation: In this practice the students will experience the easiest modulation and demodulation: the ASK. The waveform of the modulated signal will be visualized, and measurements will be made in reception, where filter measurements (central frequency and bandwidth) are learn. The envelope detector here introduced will be used in more advanced modulations.

Practice 3.- FSK modulation and demodulation (DFD): The dual filter detector -DFD- is used for FSK demodulation. This practice is also useful to measure filter parameters, and to introduce the importance of the filter transients.

Practice 4.- FSK and ASK comparison: The error probability and the required bandwidth between both modulations are experimented in the different communication channels. Students will arrive to its own conclusions on the better modulation regarding the channel disturbances.

Practice 5.- PLL tutorial: The PLL open-loop and the closed-loop behaviours are experimented, and the PLL hold-in and the capture ranges are measured. This practice is centered to know the PLL device, independently of their final use.

Practice 6.- PLL based FSK demodulation. The previous PLL study is applied to FSK demodulation. 
The PLL sensitivity, and the effect of channel perturbations in the signal jitter at the PLL input are experimented.

Practice 7.- Comparison between DFD and PLL demodulators: The error probability, the disturbance rejections and the demodulator sensitivity are compared in this practice.

Practice 8.- Carrier recovering: The carrier recover subsystem is common for the following practices devoted to phase modulation and demodulation. Here a PLL-based carrier recover is experimented and adjusted.

Practice 9.- BPSK modulation and demodulation: The different subsystems are measured, and the importance of the carrier recover adjustment is also evidenced.

Practice 10,11,12 and 13 .- DPSK, QPSK, DQPSK and QAM modulations and demodulations: In these practices the CMOS circuitry is studied, and the most significant signals are measured. In differential modulations, the dibits construction rule is deduced from oscilloscope measurements.

Practice 14.- Phase modulations comparison: In this practice all the previous experimented phase and phase-amplitude modulations are compared in bandwidth and error probability.

Practice 15.- Eye diagrams: By using base-band transmission (PCM), the effects of the channel disturbances are measured in the eye diagram. This tool is used later to experiment the quality of the different transmission channels.

Practice 16.- Constellations in phase modulations: Using the in-phase and quadrature references for BPSK modulation, the constellation of different phase and phase-amplitude modulations is experimented.

Practice 17.- Speed comparison (in bps) of the trainer modulations. Finally, the number of bits per second of the different modulations, in function of the selected transmission channel, is experimented.

\section{5.- CONCLUSIONS.}

The presented laboratory course allows the students to be familiarized with the basic concepts, the techniques and the circuits for digital communications. With the experiences, the students become trained in some bases that, in future subjects and in their professional activity, will allow them to understand the operation of the equipment that they will to repair, to maintain or to install. The course has not only learn the operation bases, but also the problems derived from the missadjustments and channel disturbances.

\section{6.- REFERENCES}

[1] Curricula in the new professional education. http://www.xtec.es/fp/

[2] Simon Haykin. "Communication Systems". John Wiley \& Sons. 1983 (2. Ed.).

[3] User manual of the EC-796 Trainer. Promax S.A.

[4] Bertran, E.; Losciuto, F. "Automatic Phase Detector for Digital Communications". Electronic Design, vol.45, n. 19. September 1997. Pp.: 168-170. 